\title{
Mapping of Neural Pathways that Influence Diaphragm Activity and Project to the Lumbar Spinal Cord in Cats
}

\author{
C.D. Rice ${ }^{1}$, S. A. Weber ${ }^{2}$, A.L. Waggoner ${ }^{2}$, M.E. Jessell ${ }^{2}$, and B. J. Yates ${ }^{1,2}$ \\ ${ }^{1}$ Department of Otolaryngology, University of Pittsburgh, Pittsburgh, PA, USA 15213 \\ 2 Department of Neuroscience, University of Pittsburgh, Pittsburgh, PA, USA 15213
}

\section{Abstract}

During breathing, the diaphragm and abdominal muscles contract out of phase. However, during other behaviors (including vomiting, postural adjustments, and locomotion) simultaneous contractions are required of the diaphragm and other muscle groups including abdominal muscles. Recent studies in cats using transneuronal tracing techniques showed that in addition to neurons in the respiratory groups, cells in the inferior and lateral vestibular nuclei (VN) and medial pontomedullary reticular formation (MRF) influence diaphragm activity. The goal of the present study was to determine if neurons in these regions have collateralized projections to both diaphragm motoneurons and the lumbar spinal cord. For this purpose, the transneuronal tracer rabies virus was injected into the diaphragm and the monosynaptic retrograde tracer Fluoro-Gold (FG) was injected into the Th13-L1 spinal segments. A large fraction of MRF and VN neurons (median of 72 and 91\%, respectively) that were infected by rabies virus were dual-labeled by FG. These data show that many MRF and VN neurons that influence diaphragm activity also have a projection to the lumbar spinal cord, and thus likely are involved in coordinating behaviors that require synchronized contractions of the diaphragm and other muscle groups.

\section{Keywords}

Vomiting; locomotion; respiration; rabies virus; transneuronal tracer

\section{Introduction}

During breathing, the diaphragm and abdominal muscles contract out of phase to move air in and out of the lungs (Feldman 1986). The connections and electrophysiological properties of the neurons that comprise the brainstem respiratory groups are appropriate to activate these muscles independently during inspiration and expiration (Feldman 1986). However, during other behaviors, simultaneous increases in the activity of both the diaphragm and other muscle groups including abdominal muscles are required; such behaviors include protective reflexes such as vomiting and postural adjustments that result in alterations in the resting length of the respiratory muscles (Yates et al. 2002). There is evidence that bulbospinal neurons in addition to those in the brainstem respiratory groups are required to coordinate these responses (Morris et al. 2003). For example, most bulbospinal inspiratory neurons are actively inhibited and mainly silent during emesis (Bianchi and Grelot 1989; Miller et al. 1990), indicating that respiratory group neurons are not responsible for eliciting vomiting-related diaphragm contractions. In addition, both neurophysiological (Woodring and Yates 1997) and lesion

Address Correspondence To: Dr. Bill Yates, University of Pittsburgh, Department of Otolaryngology, Room 519, Eye and Ear Institute, Pittsburgh, PA 15213, Phone: 1-412-647-9614, FAX: 1-412-647-0108, byates@ pitt.edu, URL: http://www.pitt.edu/ byates/yates.html. 
(Yates et al. 1995) experiments have demonstrated that respiratory group neurons are insufficient to mediate vestibular system influences on respiratory activity.

Transneuronal tracing studies have been conducted in two emetic species (ferrets and cats) to determine the locations of neurons outside of the respiratory groups that regulate respiratory muscle contractions. In ferrets, neurons in the medullary medial reticular formation (mMRF) were infected at short survival times after injection of pseudorabies virus into either the diaphragm (Yates et al. 1999) or abdominal muscles (Billig et al. 1999; Billig et al. 2001). Use of two recombinants of pseudorabies virus that could be independently detected showed that some mMRF neurons influence the activity of both the diaphragm and abdominal muscles (Billig et al. 2000). The neural circuitry providing inputs to phrenic motoneurons has also been traced in cats, by injecting the transneuronal tracer rabies virus into the diaphragm (Lois et al. 2009) as well as by placing the retrograde monosynaptic tracer horseradish peroxidase into the vicinity of diaphragm motoneurons (Rikard-Bell et al. 1984; Onai and Miura 1986). Both approaches suggested that the descending motor pathways that regulate diaphragm activity are more extensive in cats than in ferrets (Yates et al. 1999) or rodents (Dobbins and Feldman 1994), and arise from neurons in the pontine medial reticular formation (pMRF) and lateral, medial and inferior vestibular nuclei, as well as the brainstem respiratory groups and mMRF.

Prior tracing studies in felines also showed that a subset of neurons in the mMRF, pMRF, and vestibular nuclei have axons that extend to the upper lumbar spinal cord (Kuypers and Maisky 1975; Hayes and Rustioni 1981; Kausz 1991), where abdominal motoneurons are located (Feldman 1986). However, it remains to be determined whether the same neurons in these regions that influence diaphragm activity also provide projections to the lumbar spinal cord, and thus have the potential of simultaneously altering the activity of the diaphragm and other muscle groups including abdominal muscles. This was the goal of the present study, which combined injections of the retrograde monosynaptic tracer Fluoro-Gold (FG) into the lumbar spinal cord with injections of the transneuronal tracer rabies virus into the diaphragm. We tested the hypothesis that neurons in the mMRF, pMRF, and vestibular nuclei that influence diaphragm activity also have projections to the lumbar spinal cord.

\section{Methods}

Experiments were conducted on six adult cats (Liberty Research, Waverly, NY) using methodology approved by the University of Pittsburgh's Institutional Animal Care and Use Committee. Surgical procedures were conducted under isoflurane anesthesia, as described in our previous studies (Lois et al. 2009; Rice et al. 2009). Postoperative analgesia was provided by intramuscular injections of ketoprofen for 3 days following the surgery $(2 \mathrm{mg} / \mathrm{kg}$ initial dose and $1 \mathrm{mg} / \mathrm{kg}$ subsequent doses). An initial surgery was performed to inject a 3\% FG

(Fluorochrome LLC, Denver, CO) solution into the Th13-L1 spinal segments. For this purpose, the animal was secured in a stereotaxic frame, a small laminectomy was performed to expose the target segments, and the dura was incised. Since our objective was to retrogradely label all the descending projections reaching the upper lumbar cord, large injections were performed. The needle of a $1 \mu \mathrm{l}$ Hamilton syringe was inserted through the dorsal root entry zone to a depth of $2.5 \mathrm{~mm}$ with the use of a Narishige (Tokyo, Japan) manipulator, and $1 \mu \mathrm{l}$ of FG was injected over a $5 \mathrm{~min}$ interval. Subsequently, the needle was removed, and two additional injections were made on the same side, spaced $\sim 5 \mathrm{~mm}$ apart along the rostrocaudal axis. A similar series of injections was then made on the contralateral side. After all injections were complete, the muscle and skin overlying the exposed cord segments were closed using suture.

Following a recovery time of 14-21 (median of 19 days) days after the FG injections into the upper lumbar spinal cord, animals were transferred to a Biosafety Level 2 facility for injection of rabies virus into the diaphragm, using procedures identical to those in a previous study 
(Lois et al. 2009). A total volume of $200 \mu \mathrm{l}(\mathrm{n}=2), 250 \mu \mathrm{l}(\mathrm{n}=2)$, or $300 \mu \mathrm{l}(\mathrm{n}=2)$ of the $\mathrm{N} 2 \mathrm{C}$ strain of rabies virus at a titer $1 \times 10^{8}$ plaque forming units $/ \mathrm{ml}$ was injected at multiple sites across the costal region of the left diaphragm. After survival times of $97 \mathrm{~h}(\mathrm{n}=2), 100 \mathrm{~h}(\mathrm{n}=2)$, or $116-118 \mathrm{~h}(\mathrm{n}=2)$ following rabies injections into the diaphragm, animals were anesthetized using ketamine $(15 \mathrm{mg} / \mathrm{kg}$ ) and acepromazine $(1 \mathrm{mg} / \mathrm{kg})$ injected intramuscularly, followed by pentobarbital sodium $(40 \mathrm{mg} / \mathrm{kg})$ injected intraperitoneally. The animals were then perfused with 2 liters of $4 \%$ paraformaldehyde-lysine-periodate fixative, and the brainstem and spinal cord were removed, postfixed, and sectioned as discussed in a prior manuscript (Lois et al. 2009). Sections were collected sequentially in six bins of phosphate-buffered saline.

One bin of sections through the diencephalon, midbrain, brainstem, and cervical spinal cord segments were processed using avidin-biotin immunoperoxidase techniques to visualize rabies-infected neurons, as in our earlier study (Lois et al. 2009). This analysis utilized a mouse monoclonal antibody directed against the rabies virus phosphoprotein that resides in the infective nucleocapsid core (Kelly and Strick 2000). Sections through the lower thoracic and upper lumbar segments in one bin were mounted on slides, so that the presence of FG at the injection site could be verified. We relied on the endogenous fluorescence of FG to confirm that the target segments were successfully injected with the tracer. One bin of sections through the brainstem was processed using immunofluorescence techniques to dual-localize rabies and FG, using a protocol similar to that we previously employed to co-localize rabies and an enzyme involved in the production of serotonin (Rice et al. 2009). FG was identified with the use of a polyclonal rabbit anti-FG antibody (1:200, Fluorochrome LLC), whereas rabies virus was recognized using the mouse monoclonal antibody described above (1:30). Labeled cells were visualized using goat anti-rabbit secondary antibody conjugated to CY3 (1:500, Jackson ImmunoResearch Laboratories, West Grove, PA) and goat anti-mouse secondary antibody conjugated to BODIPY-FL (1:300, Molecular Probes, Eugene, OR). As such, rabies-infected cells exhibited green fluorescence, FG-labeled cells exhibited red fluorescence, and duallabeled cells appeared yellow. Examples of labeled neurons from animal C95 are shown in Fig. 1.

Immunoperoxidase sections were examined to determine the regions of the cervical spinal cord, brainstem, midbrain, and diencephalon that contained rabies infected-neurons. Immunofluorescent sections were inspected using an Olympus BX51TRF photomicroscope equipped with a Hamamatsu camera (Hamamatsu Photonics, Hamamatsu, Japan) and a Simple-32 PCI image analysis system (Compix, Lake Oswego, OR). Following an initial qualitative analysis, six corresponding representative sections from each case were selected for photography and quantitative analysis. These sections were identified using Berman's cat brainstem atlas (Berman 1968), and were located at the following levels posterior (P) to stereotaxic zero: P14.5, P11, P8.5, P7, P6, and P5. All of the brainstem regions containing rabies and FG immunopositive neurons were photographed at both low and high magnification, using epifluorescence in combination with filters that selectively excited CY3 or BODIPY-FL and in double exposures that revealed the cellular localization of both FG and rabies. Great care was taken to ascertain that yellow fluorescence reflected the colocalization of both the BODIPY-FL and CY3 fluorophors and was not attributable to the presence of overlapping cells that each contained one of the fluorophors. Montages of images were assembled using PTGuiPro photostitching software (New House Internet Services B.V., Netherlands); an example of such a montage is shown in Fig. 1E. Such montages, in conjunction with observations of sections at high magnification, were used to generate plots of the locations of labeled cells, such as those provided in Fig. 2 for animal C95. Data were tabulated and statistical analyses were performed using Prism 5 software (GraphPad Software, San Diego, CA). 


\section{Results}

FG-labeled cells were prevalent throughout the gray matter of the Th13-L1 spinal cord of all animals, showing that we had effectively made large injections of FG into these segments. As illustrated in Fig 2, FG-immunopositive cell bodies were additionally present in regions of the medulla and pons that are known to contain neurons with projections to the upper lumbar spinal cord, including the mMRF, pMRF, lateral and inferior vestibular nuclei, and caudal portions of the medial vestibular nucleus (Kuypers and Maisky 1975;Hayes and Rustioni 1981;Kausz 1991). In addition, some FG-labeled cells were observed in the ventrolateral reticular formation of the caudal medulla (e.g., the P14.5 level shown in Fig, 2), which contains ventral respiratory group neurons that regulate the activity of abdominal muscles (Feldman 1986).

The distribution of rabies-infected cells was similar to that reported in our prior manuscripts (Lois et al. 2009; Rice et al. 2009). In four of the six animals (C95, C96, C111, and C112), no infected neurons were observed in the midbrain, diencephalon or cortex, suggesting that rabies virus had passed retrogradely through only a few synapses (Lois et al. 2009). However, because a variety of factors influence the rate at which a virus passes transneuronally through neural circuitry (Lois et al. 2009), it is difficult to ascertain the exact number of synapses separating the infected brainstem neurons from phrenic motoneurons. In the other two animals (C93 and C94), rabies-infected cells were present in the midbrain and diencephalon, and brainstem labeling was much more prevalent than in the four cases with early-stage infection.

Fig. 2 illustrates the typical pattern of rabies virus immunoreactivity in the brainstem. Infected neurons were present in ventrolateral nucleus tractus solitarius and in the ventrolateral portion of the caudal medulla, in the regions known to contain dorsal and ventral respiratory group neurons (Feldman 1986;Lois et al. 2009). Infected cells were also present in large numbers in the medial, inferior, and lateral vestibular nuclei, as well as the mMRF and pMRF. For quantification, we classified the lateral reticular formation as the lateral tegmental field, and the medial reticular formation as the region comprised of the paramedian reticular nucleus and the magnocellular and gigantocellular tegmental fields (Berman 1968); these divisions are demarked in Fig. 2 by blue lines. In addition, the pons was defined as the region rostral to the P7 level in Berman's atlas (Berman 1968). We excluded raphe pallidus and raphe magnus when determining the number of labeled neurons in the medial reticular formation. A prior study showed that only limited numbers of serotoninergic neurons in each raphe nucleus were infected following rabies virus injections into the diaphragm, except in cases where viral infection had become very extensive (Rice et al. 2009). However, it was difficult to define the boundaries of raphe obscurus, and neurons located in this area were included in the counts of medial reticular formation cells.

A large fraction (median of 72\%; range of 42-99\%) of the neurons in the medial pontomedullary reticular formation that were infected by rabies virus were double-labeled for the presence of FG. The neuronal counts from each animal are provided in Table 1. A similar percentage of neurons in the mMRF (median of 71\%) and pMRF (median of 76\%) that were infected with rabies also contained FG. The presence of double labeling was also comparable in the two animals (C93 and C94) with more advanced rabies infections to that in the four cats with earlier-stage infections (see Table 1). However, only a small fraction (median of 38\%) of neurons in the medullary lateral reticular formation that were infected with rabies were colabeled with FG. Despite the limited number of cases, the fraction of rabies-infected medial and lateral reticular formation neurons that also contained FG was demonstrated to be different using a paired $\mathrm{t}$-test $(\mathrm{p}=0.03)$.

The majority of vestibular nucleus neurons (median of 91\%) that were infected with rabies virus injected into the diaphragm also were labeled for the presence of FG injected into the 
lumbar spinal cord, as indicated in Table 1. Fig. 2 illustrates that the bulk of these neurons were located in the lateral and inferior vestibular nuclei. Furthermore, a similar large fraction of vestibular nucleus neurons was double-labeled in the four cases with earlier-stage infection (median of 91\%) as in the two with more advanced infection (61 and 99\%).

\section{Discussion}

The major finding of this study is that the majority of neurons in the medial pontomedullary reticular formation and vestibular nuclei that were infected by rabies virus injected into the diaphragm were also double-labeled for the presence of FG placed in the lower thoracic and upper lumbar spinal cord. These data support the hypothesis that neurons in the medial pontomedullary reticular formation and vestibular nuclei that regulate phrenic motoneuron activity (either directly or via relay interneurons) are bulbospinal neurons that additionally make connections with cells located at more caudal spinal levels. The FG injections in this study were large, to assure that a majority of neurons with projections reaching the L1 spinal segment were labeled, such that it is unknown whether the FG-labeled cells made direct connections with motoneurons controlling abdominal musculature. However, at the very least the present data show that although neurons outside the respiratory groups provide inputs to the phrenic motor nucleus, their projections are not confined to this single motor pool and affect neuronal activity at multiple levels of the spinal cord. Previous studies showed that 50\% of lateral vestibulospinal tract neurons (Abzug et al. 1974) and 67\% of medial reticulospinal neurons (Peterson et al. 1975) supplying axonal collaterals to the cervical enlargement in cats also have descending projections to the lumbar cord, indicating that collateralized reticulospinal and vestibulospinal projections participate in coordinating forelimb and hindlimb activity. The present data suggest that vestibulospinal and reticulospinal pathways have additional functions, including producing synchronized changes in the activity of the diaphragm along with other muscle groups. In all likelihood, medial pontomedullary reticulospinal and vestibulospinal neurons that co-activate phrenic and other motoneurons play a variety of physiological roles. One prior study suggested that mMRF neurons coordinate the simultaneous discharges of the diaphragm and abdominal muscles that generate retching (Miller et al. 1996). Stimulation of vestibular afferents (Rossiter et al. 1996) or elimination of vestibular inputs through inner ear lesions (Cotter et al. 2001) produce alterations in both diaphragm and abdominal muscle activity, which could also be mediated through such collateralized projections. It is also feasible that branching projections from the mMRF and vestibular nuclei could participate in the coupling of breathing and motor activities involving limb musculature, including locomotion (Bramble and Carrier 1983; Viala et al. 1987; Kawahara et al. 1989). Additional studies will be needed to establish the physiological role of these connections, and whether distinct populations of medial pontomedullary reticular formation and vestibular nucleus neurons subserve different functions in the synchronization of diaphragm contractions with other activities.

An important consideration when interpreting the present data is whether rabies virus infected neurons other than those regulating diaphragm activity. A variety of studies have shown that rabies is selectively uptaken by motoneurons, and not by terminals of sympathetic and parasympathetic efferents or sensory afferents, when injected into skeletal muscles (Tang et al. 1999; Ugolini 2008; Lois et al. 2009). This selectivity is presumably due to the high affinity of the virus for the skeletal muscle nicotinic receptor, which concentrates rabies at the neuromuscular junction (Lafon 2005). This rapid binding to nicotinic receptors also minimizes leakage of virus from the muscle where it is placed, which allows rabies to be effectively used for tracing neural pathways regulating the activity of a particular muscle (Kelly and Strick 2000). In prior studies where similar amounts of rabies were injected into the diaphragm using the same strategy employed in the present experiments, we noted little labeling of neurons in the middle and lower thoracic spinal cord until the infection of the nervous system was 
extensive and virus had passed across many synapses (Lois et al. 2009). These findings suggest that our method for placing rabies virus into the diaphragm is not conducive to infecting nontarget motoneurons, particularly those innervating intercostal and abdominal muscles (the muscles in closest proximity to the injection site). For these reasons, it seems likely that the brainstem neurons that were immunopositive for rabies virus in this study made direct or multisynaptic connections with phrenic motoneurons.

In summary, the present data confirm and extend previous findings from experiments using transneuronal tracing techniques in cats showing that in addition to respiratory group neurons, an appreciable number of cells in the medial pontomedullary reticular formation and vestibular nuclei provide relatively direct inputs to phrenic motoneurons (Lois et al. 2009). This study showed that most medial reticular formation and vestibular nucleus neurons that regulate diaphragm activity are bulbospinal neurons, and have axonal projections that reach the lumbar spinal cord. As such, these neurons likely are involved in coordinating behaviors that require synchronized contractions of the diaphragm and other muscle groups, including musculature controlled by motoneurons located in the lumbar cord.

\section{Acknowledgments}

The authors thank Lucy Cotter, James Lois, and Derek Reighard for technical assistance, as well as Dr. Peter Strick for providing rabies virus and anti-rabies antibodies. Funding was provided by grant R01-DC003732 from the National Institutes of Health (USA). Core support was provided by grant P40-RR-018604 from the National Center for Research Resources of the National Institutes of Health.

\section{References}

Abzug C, Maeda M, Peterson BW, Wilson VJ. Cervical branching of lumbar vestibulospinal axons. With an appendix by C.P. Bean. J Physiol 1974;243:499-522. [PubMed: 4449072]

Berman, AI. The Brain Stem of the Cat. University of Wisconsin Press; Madison: 1968.

Bianchi AL, Grelot L. Converse motor output of inspiratory bulbospinal premotoneurones during vomiting. Neurosci Lett 1989;104:298-302. [PubMed: 2812545]

Billig I, Foris JM, Card JP, Yates BJ. Transneuronal tracing of neural pathways controlling an abdominal muscle, rectus abdominis, in the ferret. Brain Res 1999;820:31-44. [PubMed: 10023028]

Billig I, Foris JM, Enquist LW, Card JP, Yates BJ. Definition of neuronal circuitry controlling the activity of phrenic and abdominal motoneurons in the ferret using recombinant strains of pseudorabies virus. J Neurosci 2000;20:7446-7454. [PubMed: 11007904]

Billig I, Hartge K, Card JP, Yates BJ. Transneuronal tracing of neural pathways controlling abdominal musculature in the ferret. Brain Res 2001;912:24-32. [PubMed: 11520490]

Bramble DM, Carrier DR. Running and breathing in mammals. Science 1983;219:251-256. [PubMed: 6849136]

Cotter LA, Arendt HE, Jasko JG, Sprando C, Cass SP, Yates BJ. Effects of postural changes and vestibular lesions on diaphragm and rectus abdominis activity in awake cats. J Appl Physiol 2001;91:137-144. [PubMed: 11408424]

Dobbins EG, Feldman JL. Brainstem network controlling descending drive to phrenic motoneurons in rat. J Comp Neurol 1994;347:64-86. [PubMed: 7798382]

Feldman, JL. Neurophysiology of breathing in mammals. In: Bloom, FE., editor. Handbook of Physiology. The Nervous System, IV. Intrinsic Regulatory Systems of the Brain. American Physiological Society; Bethesda, MD: 1986. p. 463-524.

Hayes NL, Rustioni A. Descending projections from brainstem and sensorimotor cortex to spinal enlargements in the cat. Single and double retrograde tracer studies. Exp Brain Res 1981;41:89-107. [PubMed: 6162664]

Kausz M. Arrangement of neurons in the medullary reticular formation and raphe nuclei projecting to thoracic, lumbar and sacral segments of the spinal cord in the cat. Anat Embryol (Berl) 1991;183:151163. [PubMed: 2035851] 
Kawahara K, Kumagai S, Nakazono Y, Miyamoto Y. Coupling between respiratory and stepping rhythms during locomotion in decerebrate cats. J Appl Physiol 1989;67:110-115. [PubMed: 2759934]

Kelly RM, Strick PL. Rabies as a transneuronal tracer of circuits in the central nervous system. J Neurosci Meth 2000;103:63-71.

Kuypers HG, Maisky VA. Retrograde axonal transport of horseradish peroxidase from spinal cord to brain stem cell groups in the cat. Neurosci Lett 1975;1:9-14. [PubMed: 19604744]

Lafon M. Rabies virus receptors. J Neurovirol 2005;11:82-87. [PubMed: 15804965]

Lois JH, Rice CD, Yates BJ. Neural circuits controlling diaphragm function in the cat revealed by transneuronal tracing. J Appl Physiol 2009;106:138-152. [PubMed: 18974365]

Miller AD, Nonaka S, Jakus J, Yates BJ. Modulation of vomiting by the medullary midline. Brain Res 1996;737:51-58. [PubMed: 8930349]

Miller AD, Nonaka S, Lakos SF, Tan LK. Diaphragmatic and external intercostal muscle control during vomiting: behavior of inspiratory bulbospinal neurons. J Neurophysiol 1990;63:31-36. [PubMed: 2299384]

Morris KF, Baekey DM, Nuding SC, Dick TE, Shannon R, Lindsey BG. Invited review: Neural network plasticity in respiratory control. J Appl Physiol 2003;94:1242-1252. [PubMed: 12571145]

Onai T, Miura M. Projections of supraspinal structures to the phrenic motor nucleus in cats studied by a horseradish peroxidase microinjection method. J Autonom Nerv Syst 1986;16:61-77.

Peterson BW, Maunz RA, Pitts NG, Mackel RG. Patterns of projections and branching of reticulospinal neurons. Exp Brain Res 1975;23:333-351. [PubMed: 1183508]

Rice CD, Lois JH, Kerman IA, Yates BJ. Localization of serotoninergic neurons that participate in regulating diaphragm activity in the cat. Brain Res 2009;1279:71-81. [PubMed: 19433074]

Rikard-Bell GC, Bystrzycka EK, Nail BS. Brainstem projections to the phrenic nucleus: a HRP study in the cat. Brain Res Bul 1984;12:469-477.

Rossiter CD, Hayden NL, Stocker SD, Yates BJ. Changes in outflow to respiratory pump muscles produced by natural vestibular stimulation. J Neurophysiol 1996;76:3274-3284. [PubMed: 8930272]

Tang Y, Rampin O, Giuliano F, Ugolini G. Spinal and brain circuits to motoneurons of the bulbospongiosus muscle: retrograde transneuronal tracing with rabies virus. J Comp Neurol 1999;414:167-192. [PubMed: 10516590]

Ugolini G. Use of rabies virus as a transneuronal tracer of neuronal connections: implications for the understanding of rabies pathogenesis. Dev Biol (Basel) 2008;131:493-506. [PubMed: 18634512]

Viala D, Persegol L, Palisses R. Relationship between phrenic and hindlimb extensor activities during fictive locomotion. Neurosci Lett 1987;74:49-52. [PubMed: 3561874]

Woodring SF, Yates BJ. Responses of ventral respiratory group neurons of the cat to natural vestibular stimulation. Am J Physiol Regul Integr Comp Physiol 1997;273:R1946-R1956.

Yates BJ, Billig I, Cotter LA, Mori RL, Card JP. Role of the vestibular system in regulating respiratory muscle activity during movement. Clin Exp Pharmacol Physiol 2002;29:112-117. [PubMed: 11906468]

Yates BJ, Siniaia MS, Miller AD. Descending pathways necessary for vestibular influences on sympathetic and inspiratory outflow. Am J Physiol Regul Integr Comp Physiol 1995;37:R1381R1385.

Yates BJ, Smail JA, Stocker SD, Card JP. Transneuronal tracing of neural pathways controlling activity of diaphragm motoneurons in the ferret. Neurosci 1999;90:1501-1513. 


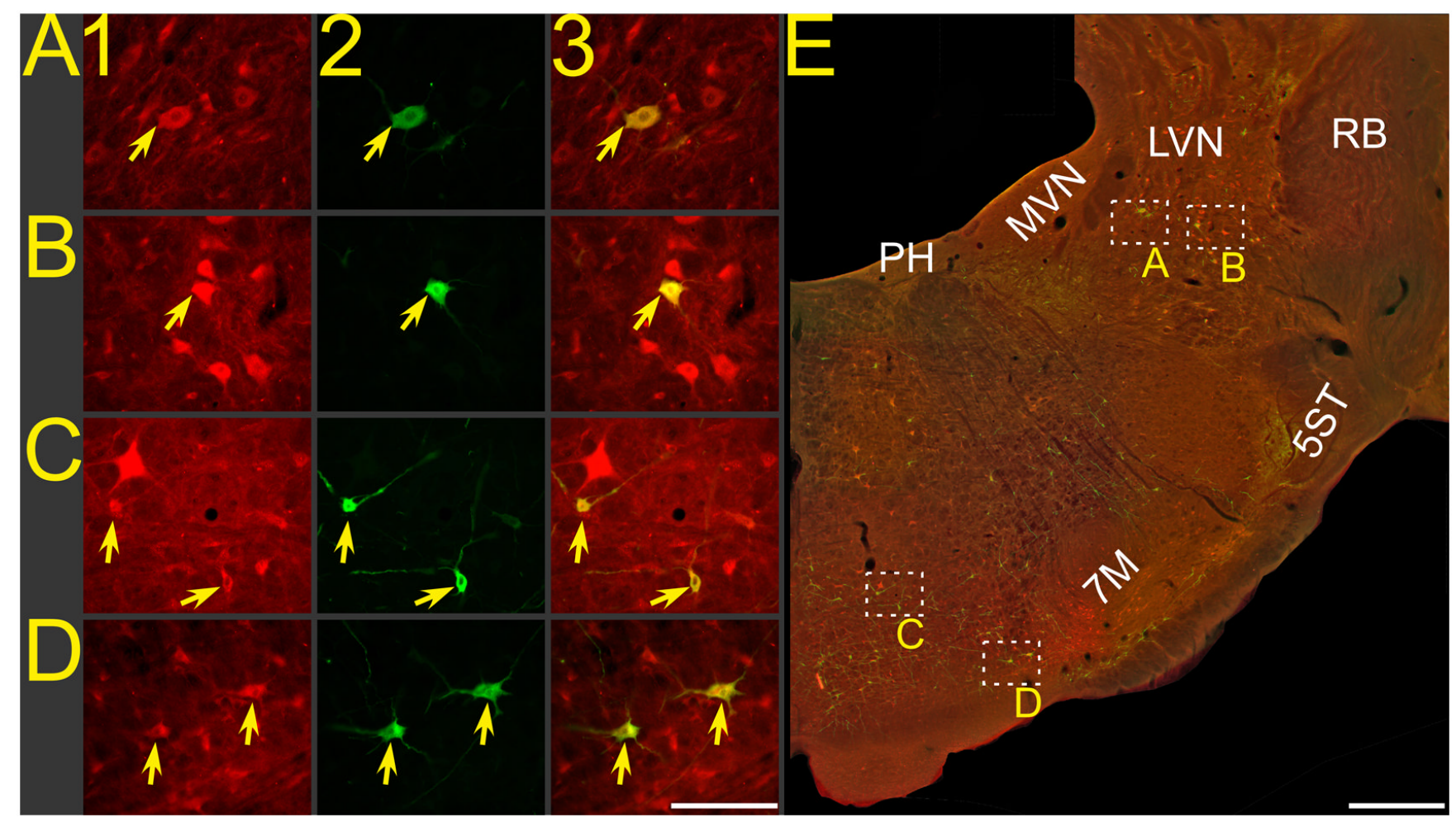

Figure 1.

Photomicrographs of neurons in animal C95, from a brainstem section $\sim 8.5 \mathrm{~mm}$ posterior to stereotaxic zero that was processed using immunofluorescence to dual-localize FG and rabies virus. A-D: photomicrographs taken at high magnification; column 1 shows FG immunopositivity, column 2 shows rabies immunopositivity, and column 3 shows the combined immunopositivity for both antigens. Arrows denote examples of double-labeled neurons. E: a montage of photomicrographs taken at lower magnification, showing immunopositivity to both rabies and FG. Boxes in panel $E$ show the locations of the neurons depicted at higher magnification in $A-D$. Calibration bars represent $100 \mu \mathrm{A}$ in $A-D$ and $1 \mathrm{~mm}$ in $E$. Abbreviations: $5 S T$, trigeminal spinal tract; $7 M$, facial motor nucleus; $L V N$, lateral vestibular nucleus; $M V N$, medial vestibular nucleus; $P H$, prepositus hypoglossi; $R B$, restiform body. 
$\mathrm{P} 14.5$

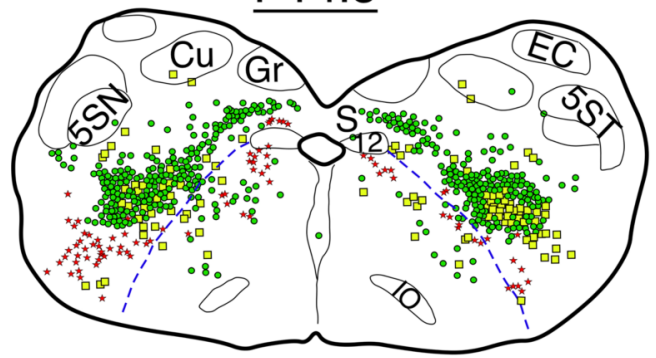

P11

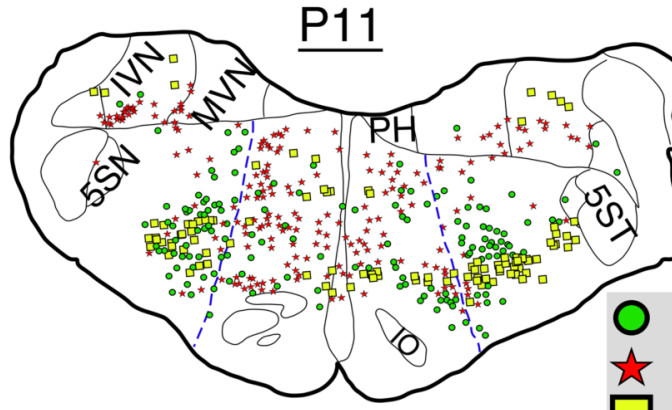

P8.5
Rabies only

FluoroGold only

Double-labeled

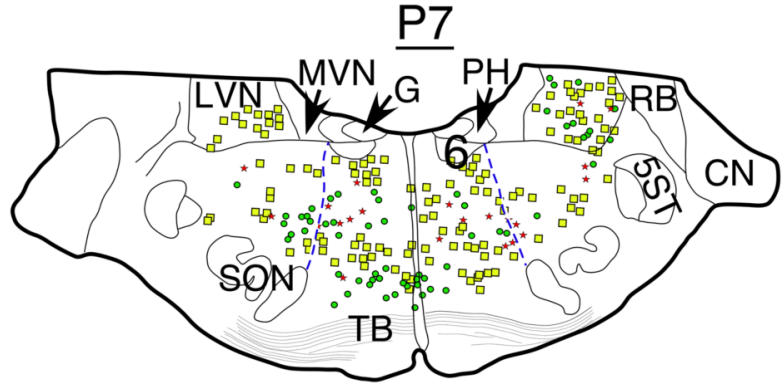

P6

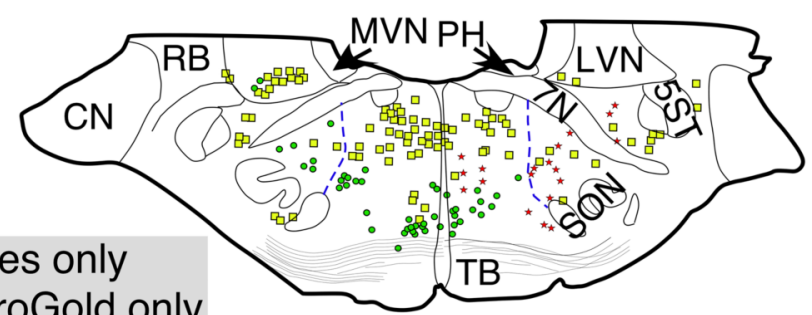

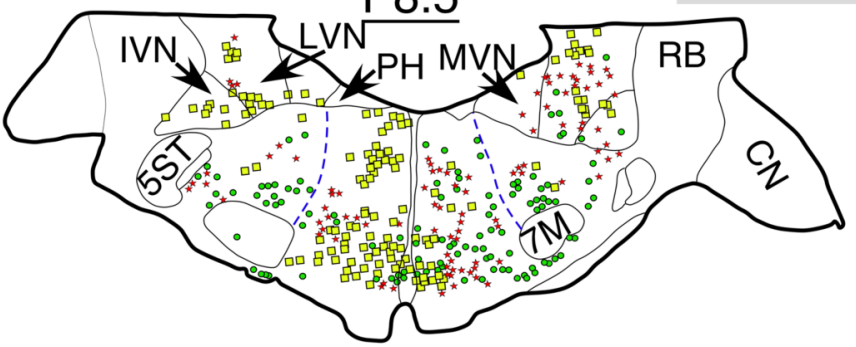

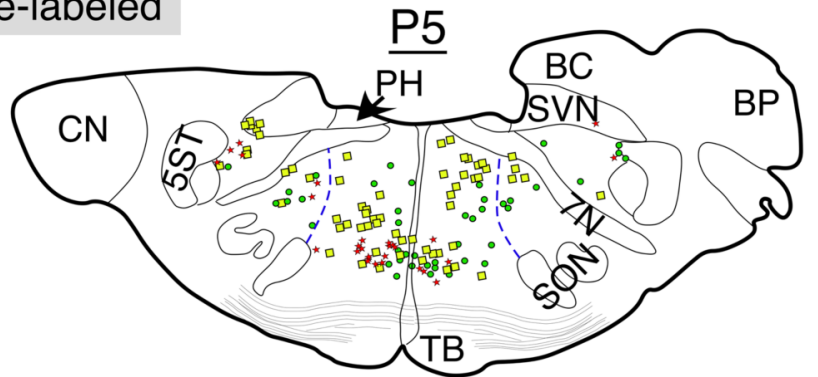

Figure 2.

Drawings of representative sections from animal C95 showing the locations of rabiesimmunopositive neurons, FG-immunopositive neurons, and neurons that were immunopositive for both FG and rabies. Regions containing labeled cells were identified using Berman's cat brainstem atlas (Berman 1968). The location of each section relative to stereotaxic zero (P0) is indicated. Blue lines in each section show the demarcation between the medial and lateral reticular formation. Abbreviations: $5 S N$, spinal trigeminal nucleus; $5 S T$, spinal trigeminal tract; 6 , abducens nucleus; $7 M$, facial motor nucleus; $7 N$, facial nerve; 12 , hypoglossal nucleus; $B C$, brachium conjunctivum; $B P$, brachium pontis; $C N$, cochlear nuclei; $C u$, cuneate nucleus; $E C$, external cuneate nucleus; $G$, genu of facial nerve; $G r$, gracile nucleus; $I O$, inferior olivary nucleus; $I V N$, inferior vestibular nucleus; $L V N$, lateral vestibular nucleus; $M V N$, medial vestibular nucleus; $P H$, prepositus hypoglossi; $R B$, restiform body; $S$, solitary nucleus; $S O N$, superior olivary nucleus; $S V N$, superior vestibular nucleus; $T B$, trapezoid body. 


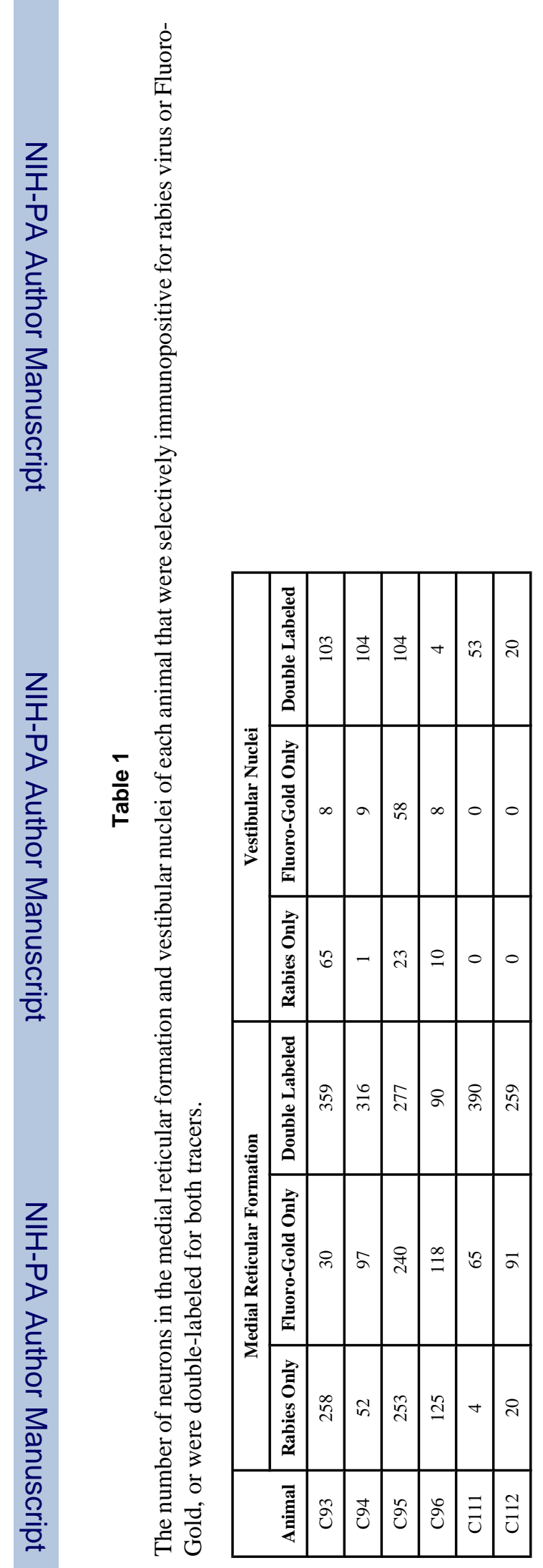

Exp Brain Res. Author manuscript; available in PMC 2011 May 1. 